\title{
OBSERVATIONAL CONSTRAINTS ON DYNAMOS IN COOL STARS
}

\author{
C.J. SCHRIJVER \\ Astronomical Institute Utrecht, P.O. Box 80000, Utrecht, The Netherlands
}

\begin{abstract}
Non-radiatively heated outer atmospheres are common among stars on the cool side of the HR diagram. These atmospheres resemble that of the Sun in which the bulk of the heating is associated with magnetic fields. Consequently it is assumed that other cool stars also generate and maintain an internal magnetic field through a dynamo action. This field gives rise to a wide spectrum of phenomena in the stellar atmosphere. The dynamo process that sustains the field thrives on the interaction of rotation and turbulent convection. In this review I formulate a set of propositions outlining our present knowledge of this interaction as distilled from stellar observations. In doing so, I outline the effects of stellar evolution on rotation rate, discuss some of the proposed "dividing lines" in the HR-diagram, and comment on the possible role of the convective turnover time in dynamo efficiency and on dynamo activity for extremely slowly and rapidly rotating stars.
\end{abstract}

\section{INTRODUCTION}

The highly structured solar atmosphere has fascinated astronomers from the moment we were aware of its existence. The classical, spherically-symmetric stellar models that were constructed in the first half of this century explained why, given the mass, the solar radius and luminosity have the observed values. The filamentary nature of the clearly non-radiatively heated chromosphere and corona, however, is still far from fully understood (see, for instance, proceedings edited by Ulmschneider et al. 1991). We do know that the solar magnetic field enables subphotospheric mechanical energy to reach the supraphotospheric plasma trapped in that field. The dissipation of that energy heats the plasma to temperatures in excess of a million Kelvin. It can be no surprise that similar processes occur in the atmospheres of stars other than the Sun. This has opened the way for testing ideas about the origin of the magnetic field, its structure, and its effects in stellar atmospheres: the dependence of activity on fundamental stellar parameters such as age, mass and radius can be analysed, while relationships with other parameters such as rotation rate and the properties of binary companions can also be studied.

The combination of solar and stellar observational material has deepened our understanding of the phenomenon that observers refer to as "atmospheric activity" while it has at the same time stimulated the development of a "dynamo theory" for the behaviour of magnetic fields in stellar interiors. In this review I summarize a selection of presently available constraints concerning stellar 
Table 1. Observational constraints on dynamo theory

1 A dynamo resulting in solar-like magnetic activity appears to operate in all rotating stars with a convection zone directly beneath the photosphere.

2 Dynamo efficiency is directly dependent on the stellar rotation rate (and only indirectly on stellar age); there is no unequivocal evidence supporting a simple scaling of the dynamo efficiency with the Rossby number.

3 The atmospheric radiative losses appear to "saturate" for stars with rotation periods below about one or two days, but it remains unclear whether this reflects a saturation in the dynamo action or merely in the heating mechanism.

4 There is some evidence that the atmospheric radiative losses associated with magnetic activity observed for main-sequence stars of a given rotation period - and by inference also the dynamo efficiency - increase with increasing depth of the convective envelope from the onset of convection up to about $B-V \approx 0.45$.

5 Dynamo action is accompanied by an effective loss of stellar angular momentum, which in all stars up to and including luminosity class III is an important process in the deceleration of rotation in addition to that expected by the structural changes in radius and internal mass distribution.

b The dividing line in the HR diagram which marks the onset of granulation - and thus of convection - in the stellar atmosphere appears to be the only such line of a fundamental physical nature where the stellar dynamo is concerned; other dividing lines delineate gradual changes (rather than fundamental ones) resulting from mass loss through the stellar magnetosphere and from changes associated with stellar evolution.

7 The presence of a companion star enhances the dynamo action in cool components of relatively close binaries not only by enforcing rapid rotation through tidal interaction, but presumably also by affecting the pattern of differential rotation resulting in an activity level well in excess of that expected from a single star of the same rotation period.

8 Activity cycles tend to increase in duration with decreasing effective temperature, while the cycle period is not a function of either rotation period or convective turnover time only.

dynamos. I do this by formulating a set of qualitative propositions (listed in Table 1) each of which is accompanied by a brief description of the evidence on which the proposition is based. The topics range from the delineation of the parameter range in which solar-like magnetic activity occurs, via the relationship between rotation and activity, to the effects of evolution and binarity.

Statements about solar-like magnetic activity in stellar interiors and atmospheres, i.e. about stellar dynamos, rely on the uniqueness of the interpretation of the diagnostics used to observe and measure stellar activity. I refer to reviews by Strassmeier (these proceedings) and Schrijver (1991) for a discussion of activity diagnostics, and concentrate on the conclusions derived from these diagnostics. First of all I define what I mean by "solar-like" in this context by formulating the quintessence of solar activity: solar activity is characterized by the existence of small (compared to the stellar surface area), long-lived (compared to photospheric convective time scales), highly structured, non-thermally heated regions above the photosphere, with plasma 
temperatures in the outer atmosphere reaching up to $1 \mathrm{MK}$ or higher. Stellar observations of spectral lines used to observe solar activity suggest solar-like activity to occur in many other stars. Evidence has even been found for the stellar equivalent of sunspots and for stellar activity cycles. Solar atmospheric activity is clearly magnetic in nature because of the close association of bright atmospheric regions with bipolar magnetic plages in the photosphere, the general appearance of bright atmospheric arches resembling the geometry of the magnetic field computed using the photospheric signal as boundary condition, and the confinement of extremely hot coronal plasma. By analogy with the Sun it is assumed that stellar activity is also magnetic in nature. Only in recent years has it become possible to measure stellar magnetic field strengths and surface filling factors more or less directly and to demonstrate the existence of a relationship between the mean value of the absolute magnetic surface flux density and atmospheric radiative losses (Saar 1991 and references therein). Schrijver (1991) combines solar and stellar data to obtain a calibration of the atmospheric radiative losses in terms of magnetic flux densities.

Knowing the relationship between atmospheric radiative losses and magnetic flux densities one can use any measure $A_{m}$ of the atmospheric radiative losses (e.g. luminosity, surface flux density, or flux normalized to the bolometric flux) - specified where necessary - as an equivalent for the level of magnetic activity. Acoustic heating processes in stars with convective envelopes also give rise to substantial chromospheric and some transition-region emission (e.g. Schrijver 1987, Rutten et al. 1991), showing up in diagrams of observed atmospheric radiative surface fluxes versus spectral colour (flux-colour diagrams) as an empirical lower limit. This non-magnetic basal contribution $A_{b}$ must be subtracted from observed radiative losses to obtain $A_{\mathrm{m}}$.

\section{CONVECTION AND ACTIVITY}

The large body of observational material now available allows the formulation of proposition number 1: "A dynamo resulting in solar-like magnetic activity appears to operate in all rotating stars with a convection zone directly beneath the photosphere." The region in the HR-diagram in which stars exhibit solar-like activity consequently lies redward of the line at which a convection zone develops immediately beneath the stellar photosphere (cf. Fig. 1).

Although proposition 1 sppears trivial in the light of current knowledge, it does put several significant constraints on dynamo theory. First of all it implies that dynamo action occurs even in stars with very shallow convective envelopes (further discussed in Section V). Secondly, it implies that dynamos also operate in the fully convective M-type dwarf stars (e.g. Giampapa and Liebert 1986). Hence, the existence of a discontinuity at the interface of the radiative interior and the convective envelope may be an important facet of dynamo theory, but its absence does not preclude dynamo action. Little is known presently about the dependence of activity on rotation for these very cool stars, although Giampapa and Liebert (1986) note that non-emission line late M-type dwarfs rotate slowly as compared to emission-line stars. Indications exist that the structure of the outer atmosphere of dwarf stars cooler than about M0 differs substantially from that in F-, G-, and K-type stars, particularly where the lower chromosphere is concerned (Rutten et al. 1989).

Activity also occurs in the young $\mathrm{T}$ Tauri stars (see, for instance, the review by Basri 1987a). It appears that when stars become visible through 

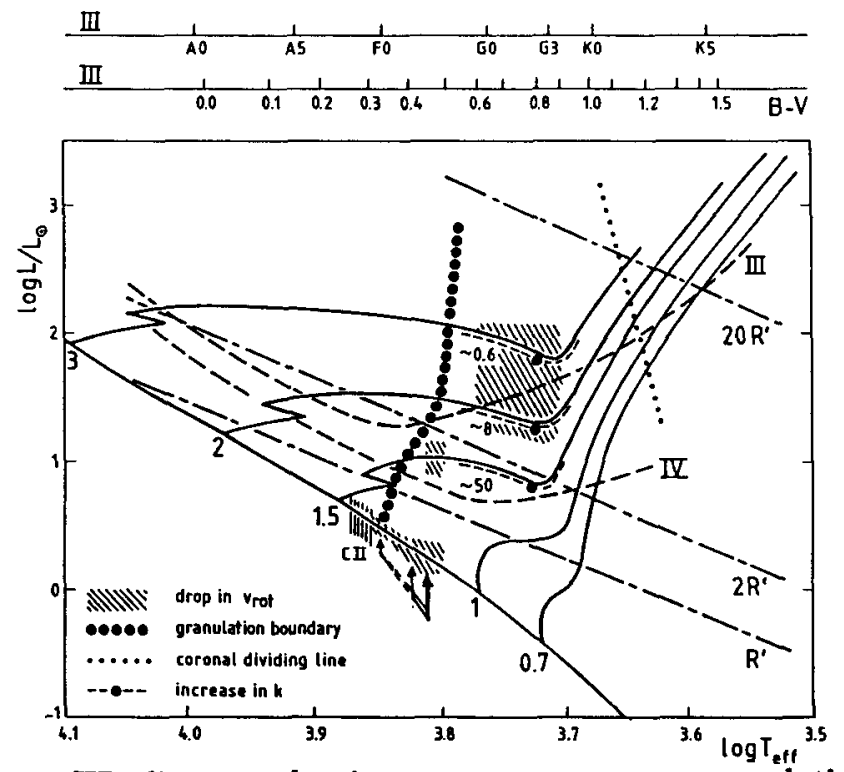

Fig. 1 HR diagram showing post main-sequence evolution (modified after Rutten and Pylyser (1988)). Stellar masses (in solar units) are given along the main sequence. Luminosity classes are delineated by long-dashed curves. The approximate position of the "granulation boundary" where the reversal in the asymmetry of photospheric lines is used as an approximate delineation of the onset of convection is marked by heavy dots (after Gray and Nagel 1989). For main-sequence stars the drop in rotational velocities (from Gray 1991) sets in close to the granulation boundary. For dwarf stars blueward of $B-V \approx 0.45$ (marked by a large arrow) the dynamo efficiency has been suggested to decrease down to $B-V \approx 0.40$ (medium-sized arrow), and possibly even down to $B-V \approx 0.3$ (small arrow; cf. Section $V$ ). Between $B-V=0.25$ and 0.29 the chromospheric C II emission, presumably reflecting largely acoustic heating in that range, increases rapidly in strength (Simon and Landsman 1991). The rotational velocities of evolving stars drop markedly within the dashed regions (cf. Rutten and Pylyser 1988). For giants of about 2 to 3 solar masses this decrease coincides with an increase in the moment of inertia associated with an internal redistribution of mass (causing a change in the gyration radius $k R$ without a substantial change in the stellar radius $R$ ), occurring in the intervals marked by dashed lines along the tracks (the point of steepest change in $k$ as a function of $T_{\text {eff }}$ is marked by a dot on the dashed lines). For the less massive subgiants the drop in velocities occurs near or immediately beyond the onset of surface convection. In all cases the rotation rates have dropped substantially prior to climbing the red giant branch preceding core-helium ignition. The numbers close to the evolutionary tracks give the approximate time intervals (in $\mathrm{Myr}$ ) in which a star evolves from the granulation boundary to the upturn onto the giant branch (data from Maeder and Meynet 1988). Stars reach the coronal dividing line (dotted; from Linsky 1992) - first proposed by Linsky and Haisch (1979) - after about a fifteenfold increase in radius since leaving the ZAMS (lines of constant radius relative to an arbitrarily chosen $R^{\prime}$ are dashed-dotted). 
the surrounding primordial cloud, they already exhibit very strong magnetic activity. This emission decreases rapidly as the stars age (cf. Section IV), but their chromospheric and coronal fluxes are indistinguishable from those of most active main-sequence and evolved stars (e.g. Walter et al. 1988). The picture is simple only for the so-called "naked T-Tauri stars" (cf. Walter 1987), however, because in other $\mathrm{T}$ Tau stars the emission from circumstellar material arranged in bipolar outflows and equatorial disks contributes strongly to, or even dominates, the stellar emission proper (e.g. Lemmens et al. 1992).

\section{DYNAMO STRENGTH AND ROTATION}

Rotation rate and activity are clearly related: when comparing any measure of stellar magnetic activity $A_{\mathrm{m}}$ with, for instance, rotation period $P_{\text {rot }}$ for stars of similar colour (Fig. $2 \mathrm{a}$ ), one sees that $A_{\mathrm{m}}$ decreases monotonically with increasing $P_{\text {rot }}$ for stars with $P_{\text {rot }} \gtrsim 1$ day (cf. Section IV). That this is a direct physical relationship in which age plays a role only through the magnetic braking of stars (see Section VI) was already implied by Skumanich (1972) and further discussed by Middelkoop and Zwaan (1981), who pointed out that while old, single stars are inactive, old binaries that are tidally synchronized are very active (cf. Section VIII).

Proposition 1 implies that a dynamo process operates whenever a star rotates, thereby excluding the possibility that there is some minimally required rate of rotation to excite the dynamo. This conclusion is, admittedly, crucially dependent on the accuracy and validity of extrapolations of the relationship between rotation and activity. Schrijver et al. (1989), for instance, argue that extrapolation of this relationship for dwarfs suggests that stellar rotation vanishes at or very near the empirical lower-limit flux in a flux-colour diagram. In their case the main activity diagnostic was the $\mathrm{Mt}$. Wilson $\mathrm{Ca} I I \mathrm{H}+\mathrm{K}$ flux, in which the lower-limit flux incorporates both the basal line-core component, probably measuring purely acoustic heating, and a line-wing component. They also pointed out that the long-term $\mathrm{Ca} I I \mathrm{H}+\mathrm{K}$ variability as well as the coronal soft X-ray emission disappear at or very near this lower-limit flux. Hence they conclude that it is unlikely that a solar-like cyclic dynamo operates in stars at the level of the $\mathrm{Ca}$ II $\mathrm{H}+\mathrm{K}$ lower limit (in agreement with the explanation of basal heating as acoustic in nature, although the convection zone of a nonrotating star may in principle be capable of generating a weak background magnetic field which plays a role in the basal heating process), and conversely that activity $A_{m}$ increases smoothly and monotonically with decreasing rotation period $P_{\text {rot }}$ (at least for $P_{\text {rot }} \geq 1$ day, see Section IV). Verifying whether there is a minimally required non-zero rotation velocity $v_{c}$ for a dynamo to operate is more difficult for single giants, because of their generally low $v \sin (i)$ values lying near the detection limit. Observed $v \sin (i)$ values set an upper limit to $v_{c}$ of about 1-2 km/s (Gray 1989a). Flux-colour diagrams also contain valuable information: while for dwarf stars one might argue that they may not spin down sufficiently to reach $v_{c}$, evolving single giant stars continue to slow down as long as their moment of inertia increases (cf. Section VI). Thus we can conclude that if the activity would discontinuously change from $A_{m}\left(v_{c}+\epsilon, T_{\text {eff }}, \ldots\right)>0$ to $A_{m}\left(v_{c}-\epsilon, T_{e x}, \ldots\right)=0$ (for small $\epsilon$ ), the evolution of the moment of inertia should yield stars on the cool side of flux-colour diagrams at the basal level, separated from the "active" stars by a gap. Such a gap is not observed so that if $v_{c} \neq 0$, $A_{\mathrm{m}}\left(v_{c}+c, T_{\text {eff }}, \ldots\right)$ is at most small compared to $A_{\mathrm{b}}$. 

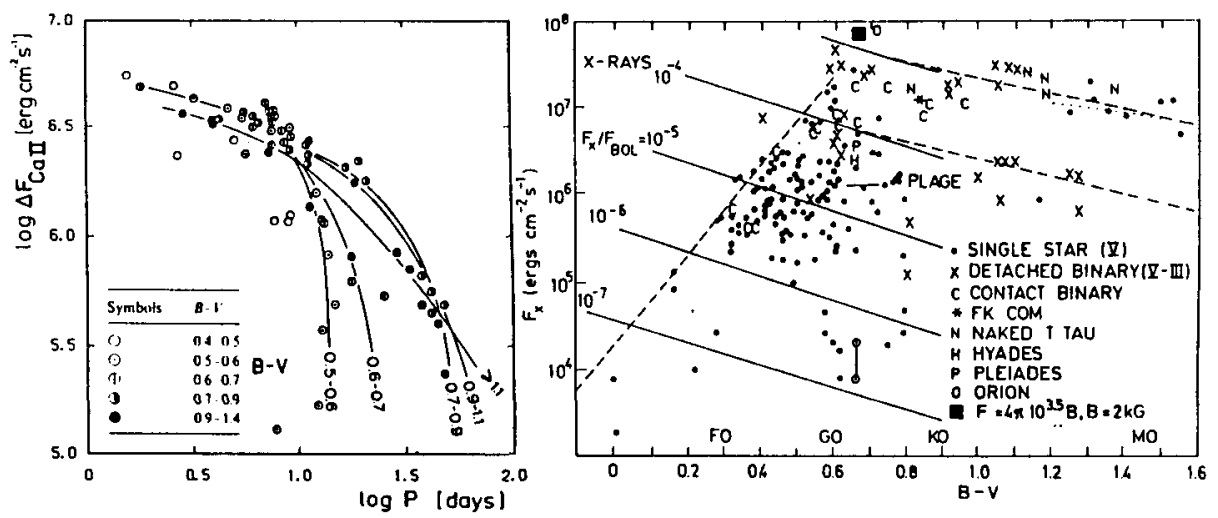

Fig. 2 a) (Left) The chromospheric $\mathrm{Ca}$ II H+K excess flux density, measuring magnetic activity, versus the stellar surface rotation period, for dwarfs in different colour intervals (from Rutten 1987). b) (Right) The soft $\mathrm{X}$-ray surface flux density $(0.2-4 \mathrm{keV}$ ) vs. colour (after Vilhu 1986).

Theoretical work of Durney and Latour (1978) suggested that there should be a relationship between activity $A_{\mathrm{m}}$ and the ratio $R_{0}$ of the rotation period $P_{\text {rot }}$ and the so-called convective-turnover time $\tau_{\mathrm{c}}$ (the ratio of a typical length scale and velocity of the convection). This ratio $R_{0}$ is known as the Rossby number which measures the influence of Coriolis forces on convective flows. Moreover, Noyes et al. (1984a) showed that $R_{0}$ is also related to the dynamo number $N_{D}$ (cf. Parker 1979): $N_{\mathrm{D}} \approx R_{\mathrm{o}}^{-2}$. Noyes (1983) and Noyes et al. (1984a) argued that the observational material might support such a relationship between $A_{m}$ and $\dot{R}_{0}$. They find the best relationship for their sample of dwarf stars by using the ratio of chromospheric $\mathrm{Ca} I I \mathrm{H}+\mathrm{K}$ flux to the bolometric flux as activity measure and the Rossby number $P_{\text {rot }} / \tau_{c}$ computed for the bottom of the convective envelope for a mixing length of 1.9 pressure scale heights for stars with $B-V \in[0.5,0.9]$, while assuming a constant turnover time for cooler stars (an assumption that is too crude, see Gilliland 1985a).

The argumentation supporting the role of $\tau_{c}$ in rotation-activity relationships stands or falls with the independence of the shape of the rotationactivity relationship on spectral type. Rutten and Schrijver (1987) argue that the data for dwarfs suggest that the shape of the rotation-activity relationship is in fact colour dependent (Fig. 2a), so that no colour-dependent scaling of either rotation period or activity level, or both, can yield a simple, colour-independent relationship between rotation rate and atmospheric radiative losses. In parallel studies, Basri (1986) and Simon and Fekel (1987) pointed out that no advantage is gained by the use of the Rossby number over the comparison of $\mathrm{Ca} \mathrm{II} \mathrm{H}+\mathrm{K}$ surface flux density and rotation period.

The defence for the relevance of a $\tau_{c}-8 c a l i n g$ becomes even more problematic when giants are included: while Rutten (1987) argues that dwarfs and single giants of the same colour and rotation period are equally active, the values of $\tau_{c}$ for evolved stars differ from those of dwarfs (Gilliland 1985a,b, but see Basri $1987 \mathrm{~b}$ for comments on the effective temperature scale used by Gilliland) so that a scaling with $\tau_{c}$ would in fact disrupt relationships. Basri (1987b), instead, noted a slight improvement of the overlap of dwarf and giant data when using 
$R_{0}$, but his data included evolved binaries which appear to behave differently from single stars (cf. Section VIII). Moreover, he notes that the relationships for each of the subclasses is somewhat worse when using Rossby numbers.

These results can at best be summarized by proposition 2: "Dynamo efficiency is directly dependent on the stellar rotation rate (and only indirectly on stellar age); there is no unequivocal evidence supporting a simple scaling of the dynamo efficiency with the Rossby number." Apparently the relationship between rotation and activity in single stars involves at least the rotation rate and a basic stellar parameter such as the effective temperature (possibly indirectly related to $\tau_{c}$ ). The functional dependence on stellar effective temperature does not allow for a simple dependence on the dimensionless Rossby number, thereby ruling out a single-parameter description $A_{\mathrm{m}}\left(R_{\mathrm{o}}\right)$, nor does it allow the rotation-activity relationship to be written as a product of functions of rotation rate and of basic stellar parameters only.

\section{RAPIDLY ROTATING STARS}

Flux-colour diagrams such as in Fig. $2 b$ and rotation-activity diagrams including young, rapidly rotating dwarf stars support proposition 3: "The atmospheric radiative losses appear to "saturate" for stars with rotation periods below about one or two days, but it remains unclear whether this reflects a saturation in the dynamo action or merely in the heating mechanism." Radiative losses in the short-period W UMa binaries in fact appear to decrease with increasing rotation rate for rotation periods below about one day (e.g. Rutten and Schrijver 1987).

Vilhu (1987) and Vilhu et al. (1989) point out that the subphotospheric mechanical fluxes $F_{\mathrm{m}}$, estimated from a mixing length model to be $\approx 1-5 \%$ of the bolometric luminosity at one pressure scale height beneath the photosphere, lie between a factor of only 3 to 30 (increasing with effective temperature) above the estimated radiative losses from the outer atmosphere for stars at "saturation." They argue that $F_{\mathrm{m}}$ limits the observed radiative losses: even if the surface filling factor of the magnetic flux (close to unity for these very active stars, e.g. Saar 1991) could still increase, it could not channel more energy from (below) the photosphere into the atmosphere.

Indirect support for an actual "saturation" of the dynamo activity and not just of the atmospheric radiative losses comes from the relationship between stellar age and activity for single main-sequence stars. Magnetic activity appears to be ubuiquitously accompanied by loss of stellar angular momentum (see Section VI). This results, for instance, in a rapid decrease and convergence of rotational velocities between the ages of the $\alpha$ Persei and Hyades clusters. MacGregor and Brenner (1991) study the rotational evolution of solar-type stars on the main sequence by modelling stars as consisting of two rigidly rotating parts, the radiative interior and the convective envelope, between which angular momentum is transferred on a time scale $\tau_{c e}$. Angular momentum loss is assumed to occur on a time scale $\tau_{J}=3 / 2 I_{\text {conv }} /\left(r_{\mathrm{A}}^{2} \dot{M}\right)$, (with $I_{\text {conr }}$ the moment of inertia of the convective envelope, $r_{\Lambda}$ the Alfvén radius, and $\dot{M}$ the mass-loss rate) consistent with a monopolar geometry for the poloidal magnetic field component. They find good agreement with the observed rotation rates for solar-type stars in the young $\alpha$ Per, Pleiades, and Hyades clusters and present surface and core rotation rates of the Sun - which differ little according to helioseismological 
observations, with a possible exception for the innermost regions - for a timeindependent core-envelope coupling time $\tau_{\mathrm{ce}} \approx 10 \mathrm{Myr}$ - about equal to the initial spin-down time - and a mean magnetic flux density that is linearly dependent on $1 / P_{\text {rot }}$ for slow rotators $\left(P_{\text {rot }} \geq 3\right.$ days $)$, but independent of the surface rotation period $P_{\text {rot }}$ for more rapid rotators, suggesting a saturation of the dynamo.

\section{THE ONSET OF ACTIVITY}

The fourth proposition addresses the onset of activity at the boundary between stars with and without convective envelopes in the HR-diagram (cf. Fig. 1): "There is some evidence that the atmospheric radiative losses associated with magnetic activity observed for main-sequence stars of a given rotation period - and by inference also the dynamo efficiency - increase with increasing depth of the convective envelope from the onset of convection up to about $B-V \approx 0.45$." Indirect evidence for the activation of a weak dynamo immediately blueward of the granulation boundary at about F0 on the main sequence is found in the decrease of the mean rotational velocities from that point onward (e.g. Gray 1989b), while in hotter stars the mean rotational velocity is a colourindependent fraction of the rotational breakup velocity (Kawaler 1987). Further indirect evidence is found in the increase of the range in X-ray emissions observed to occur from F0 onward (cf. Fig. 2b). Direct evidence supporting proposition 4 so far comes from a single paper by Walter and Schrijver (1987), which has been contested recently by Simon and Landsman (1991; see also Simon and Drake 1989). Simon and Landsman use the upper chromospheric C II emission lines to measure stellar activity. They argue that the $\mathrm{C}$ II emission of stars earlier than spectral type $F 5(B-V \approx 0.45)$ is uncorrelated with rotation. A rotationactivity relationship in these stars is hard to establish accurately, because of the relatively strong contribution of the non-magnetic basal emission $A_{\mathrm{b}}$, which Simon and Landsman do not correct for. Proper correction of the C II fluxes in their sample for the relatively strong basal flux suggests that there is indeed a relationship between rotation and activity down to at least $B-V \approx 0.40$, but that the emission level is suppressed as compared to stars of later spectral type at a given rotation period. Substantiating the claim by Walter and Schrijver (1987) that the rotation-activity relationship extends to even hotter stars requires a more extensive sample with accurate flux measurements.

Until such an extended study is completed, the hypothesis formulated by Simon and Drake (1989) and Simon and Landsman (1991) that the apparent deficiency in soft X-rays as compared to the C II and C IV emission for late-A and early-F type stars signals a strong coronal wind also remains unproven: subtraction of the relatively strong basal chromospheric flux appears to place F-type stars on or very close to the relationship between soft X-rays and C II emission defined by cooler stars, suggesting a similar atmospheric structure.

For single stars later than mid-F the dynamo efficiency appears to be independent of spectral type for stars rotating faster than about 10 days, while a colour-dependence shows up for slower rotators (Fig. 2a).

\section{ANGULAR MOMENTUM LOSS BY MAGNETIC BRAKING}

The next proposition addresses a problem related to the large-scale geometry of the magnetic field and the flow of plasma along field lines open to the interstellar space: a stellar wind blowing in a magnetosphere exerts a torque on the star, draining its angular momentum and thus applying a brake to the stellar 
rotation. Specifically: "Dynamo action is accompanied by an effective loss of stellar angular momentum, which in all stars up to and including luminosity class III is an important process in the deceleration of rotation in addition to that expected by the structural changes in radius and internal mass distribution." (Proposition 5) The rotation-activity-age relationship of main-sequence stars was touched upon in Sections $I V$ and V, while the fundamental importance of magnetic braking for binaries is discussed in Section VIII. Here I concentrate on the angular momentum loss of stars with masses up to about $3 M_{\odot}$ evolving off the main sequence towards the red giant branch (the strong activity of more massive stars in the giant phase is discussed by, e.g., Pasquini et al. 1990).

Main-sequence stars with convective envelopes (i.e. for $M \leqslant 1.3 M_{\odot}$ ) lose a substantial fraction of their angular momentum during their main-sequence phase (e.g. Soderblom et al. 1991). More massive stars, in contrast, do not develop a solar-like dynamo untill they cross the "granulation boundary" (Fig. 1), and hence retain (most of) their angular momentum until that phase. To what extent the decrease in rotation rate after the onset of convection is affected by angular momentum loss in addition to evolutionary changes in the stellar structure depends on the time scale of the angular momentum loss relative to the time scale of stellar evolution. What is known about this?

Signatures of photospheric granulation first occur around spectral type F5 for luminosity class III stars evolving through the HR-diagram, but there is no substantial drop in rotational velocities up to about $\mathrm{G} 0$. Within a few subclasses in spectral type the rotational velocities then decrease by typically over an order of magnitude. Rutten and Pylyser (1988) demonstrate that, under the assumption that stars rotates rigidly, the observed change in the mean rotational velocity of giants as a function of spectral colour is in fair agreement with that expected from the change in moment of inertia, $I$. From this they argue that the change in $I$ is the dominant process in slowing down these stars. Note that the increase in the radius of gyration $k R$ which results in the drop in rotational velocities is caused by the increase in the constant $k$ with no substantial change in radius $R$ in that part of the evolution (cf. Fig. 1).

The drop in rotational velocities is even more pronounced for the less massive subgiants, occuring not too much blueward from the onset of convection (cf. Fig. 1). Rutten and Pylyser (1988) argue that magnetic braking is the dominant factor in slowing down these stars, because the drop occurs well before a significant change in $I$. Moreover the brake has a much longer time to affect these stars: subgiants of $1.5 \mathrm{M}_{\odot}$ take some fifty million years to pass from the granulation boundary to the red giant branch, which is much longer than the roughly one and ten million years it takes $3 \mathrm{M}_{\odot}$ or $2 \mathrm{M}_{\odot}$ stars, respectively. Note that if the core-envelope synchronization time scale of $\tau_{\mathrm{co}} \approx 10 \mathrm{Myr}$ found for the main-sequence rotational evolution (cf. Section IV) is of the proper order of magnitude for these stars, then the evolution of subgiants may be slow enough for the entire star to be spun down and not just the convective envelope, while $2 M_{\odot}$ stars may only lose angular momentum from the convective envelope. Whether or not this is the case, shortly after the (sub)giants begin their ascent of the red giant branch, the convective envelope reaches down to its deepest point, encompassing about three quarters of the stellar mass (e.g. Maeder and Meynet 1989; see also Böhm-Vitense (1992) on the observed increase in the $\mathrm{N} / \mathrm{C}$ abundance ratio which is caused by the deepening of the convection zone, and which occurs after the drop in $v$ sin(i)) so that by then the bulk of the star 
exhibits at most some form of differential rotation. Consequently, dredging up angular momentum from a possibly rapidly spinning stellar core is unlikely to spin up stars as they move up along the red giant branch. The assumption of rigid rotation made by Rutten and Pylyser (1988) may change the precise way in which giants spin down prior to the red giant branch, but the magnitude of the rotation they compute for stars at the upturn onto the giant branch sets an upper limit to actual rotation rates (cf. Section VII).

Gray (e.g. 1989a, 1991) argues that the drop in $v \sin (i)$ for giants is too fast to be compatible with only structural evolutionary changes as computed by Rutten and Pylyser. I believe this criticism is largely based on the use by Gray of the intrinsically inaccurate and somewhat ambiguously plotted scale indicating spectral types rather than the colour scale in the key figure of Rutten and Pylyser (their Fig. 2a). Using Gray's (1989b) calibration of colour and spectral type, the computed drop in velocities indeed occurs within a few spectral subclasses (cf. Fig. 1) for the giants, in better agreement with Gray's data.

Gray (1989a) presents a compelling argument, however, in favour of the role of magnetic braking for LC III stars, which is based on the distribution function, $H\left(v_{\text {rot }}, B-V\right)$, of observed rotational velocities. Stars of sufficiently low masses evolve away from the main sequence towards the red giant branch moving monotonically towards later spectral type. Hence the observed population of such stars at any colour stems from a similar popolation at higher effective temperatures. If no changes in the initial $H\left(v_{\text {rot }}, B-V\right)$ and orientations of rotation axes occur as a function of time, and provided that selection effects associated with the intrinsically low rotational velocities of cool giants can be neglected, the behaviour of an evolving star can be studied by comparing properties of stars at different colours. In this respect Gray notes that $H\left(v_{\text {rat }}, B-V\right)$ changes in shape as a function of $B-V$ from what can be approximated by a Maxwell-Boltzmann distribution for stars blueward of the granulation boundary to one with a reversed asymmetry in which the rotational velocity at which the peak in $H\left(v_{\text {rot }}, B-V\right)$ occurs lies at higher values than the mean velocity for stars redward of the granulation boundary. If the changing moment of inertia $I$ were the only effect to play a role, then this would reduce the rotational velocities in time, but $H\left(v_{\text {rot }}, B-V\right)$ would not change its relative shape. Consequently, Gray argues, magnetic braking must be important. In fact he goes one step beyond this: he notes that the observed $H\left(v_{\text {rot }}, B-V\right)$ for stars beyond the strong drop in velocities is compatible with all stars of a given spectral type having the same rotation velocity, with different inclination angles of the axes of rotation and some obervational scatter smearing it to the observed curve. On this he bases his rotostat mechanism (discussed below).

Gray's interpretation is, however, not unique. A similar change in the shape of $H\left(v_{\text {sot }}, B-V\right)$ could be brought about by a differential braking with $\left|\dot{P}_{\text {rot }} / P_{\text {rot }}\right|$ decreasing with increasing $\dot{P}_{\text {rot }}$ in addition to evolutionary changes. This would result in a non-linear transformation of initial velocities before the drop in velocity into velocities beyond it. One can show that for the observed distribution functions the transformation can be approximated by a power law relating the velocities at time $t_{1}$, when the stars lie between F0 and G0, to those at time $t_{2}$, when the stars lie between G3 and K2 (two intervals, used by Gray $1989 \mathrm{a}$, on either side of the marked decrease in rotation rate): $v\left(t_{2}\right)=\alpha v\left(t_{1}\right)^{\beta}$, with $\beta \approx 0.56$. 
Can the change in shape of $H\left(v_{\text {rot }}, B-V\right)$ across the "rotational divide" mask a dependence on stellar mass, perhaps in addition to a dependence of the braking time scale on rotation rate? There is in fact a weak mass dependence in velocities blueward of the "rotation dividing line": less massive stars rotate on average less rapidly than more massive stars. Reliable distance estimates for single giants are rare, particularly for the stars redward of the divide, so that the consequences of mass-dependent effects cannot be explored further at present. On heuristic arguments, however, one may expect less massive stars to brake more strongly because of their smaller moment of inertia and their slower evolution compared to the heavier stars, while more massive stars slow down a little more than their less massive counterparts because of a somewhat larger increase in radius. Hence, the mass-dependent effects may largely cancel and thus not affect the shape of $H\left(v_{\text {zot }}, B-V\right)$ for giants too much across the "rotational divide," leaving Gray's argument favouring magnetic braking valid.

Gray argues that all giants of a given colour rotate equally fast beyond the drop in velocities (the "rotation dividing line"). He suggests the fundamental cause of this to be the so-called "rotostat" mechanism in which magnetic activity supposedly disappears once the rotation velocity drops below a colourdependent minimum. This implies that there should be a non-zero rotation velocity $v_{c}(B-V)$ at which chromospheric emissions such as the $\mathrm{Ca} \Pi \mathrm{H}+\mathrm{K}$ flux density should not differ substantially from the minimal basal value. This effect is hard to prove or disprove, because the level of the basal flux density is not known with sufficient accuracy, but. with the above arguments it is clear that Gray's arguments concerning the rotational evolution of giants and concerning the rotostat certainly do not result in a unique interpretation of the data for giant stars. The alternative interpretation, combining evolutionary effects with activity-dependent magnetic braking, is equally compatible with the data. The latter moreover yields a fair prediction of the observed decrease of rotational velocities throughout the evolution of giants. Additional evidence against the idea of a minimally required velocity to start the dynamo in dwarf stars as well as in giant stars was discussed in Section III.

\section{DIVIDING LINES}

Several so-called "dividing lines" have been suggested as boundaries in the HR-diagram between subgroups of magnetically active stars. The "rotation dividing line" proposed by Gray (1991, and references therein) most likely delineates the combined effect of magnetic braking and evolutionary changes, as discussed in Section V.I, rather than a fundamental change in the dynamo. Another well-known boundary is the "coronal dividing line" proposed by Linsky and Haisch (1979). The coronal emission from single stars redward of the dividing line is very weak (Haisch et al. 1991, for instance, quote an upper limit of $L_{X}$ of $310^{25} \mathrm{erg} / \mathrm{sec}$ for Arcturus from ROSAT observations, or less than 0.7 ergs $\mathrm{cm}^{-2} \mathrm{~s}^{-1}$, cf. Fig. 2b). The suggested causes for this dividing line include destabilization of coronal loops in the reduced gravity (e.g., Antiochos 1987) and a change in the field geometry allowing massive winds but no strong coronal emission. Recently, however, Haisch et al. $(1990,1991)$ argue that a decrease in rotation is responsible for the dividing line. Note that stars of masses between $0.7 \mathrm{M}_{\odot}$ and $3 \mathrm{M}_{\odot}$ experience about a fifteenfold increase in radius as compared to their ZAMS values upon reaching the dividing line (cf. Fig. 1). 
The observed chromospheric emission from stars beyond the coronal dividing line appears to be predominantly basal (acoustic) in nature. Judge and Stencel (1991), for instance, show that ultraviolet radiation losses from giants between spectral types K1 III and M5 III (mostly first giant branch stars) decline with effective temperature, with fluxes crowding against the extrapolation of basal fluxes found for $\mathrm{Mg}$ II $\mathrm{h}+\mathrm{k}$ by Schrijver (1987). Later spectral types (mostly second ascent or asymptotic giant branch stars) exhibit a wider range in values which Judge and Stencel suggest is related to the increased variability of these stars. Estimated wind energy fluxes are similar for the sample of first or second ascent stars, somewhat exceeding $\approx 10^{-6} F_{\text {bol }}$. Because of the decreasing basal flux with decreasing effective temperature and the more or less constant wind energy flux, the energy in the wind increases in relative importance, exceeding the chromospheric radiative energy losses for stars cooler than about 3000K (around M5 III, i.e. at the edge of the range of Fig. 1).

In conlusion (proposition 6): "The dividing line in the $\mathrm{HR}$ diagram which marks the onset of granulation - and thus of convection - in the stellar atmosphere appears to be the only such line of a fundamental physical nature where the stellar dynamo is concerned; other dividing lines delineate gradual changes (rather than fundamental ones) resulting from mass loss through the stellar magnetosphere and from changes associated with stellar evolution."

\section{ACTIVITY IN CLOSE BINARIES}

The combination of angular momentum loss in magnetically active stars and tidal interaction in binaries has the interesting result that ageing stars can actually be spun up, increasing their rotational angular momentum at the expense of the orbital angular momentum. As a consequence the orbit becomes tighter (which may eventually lead to coalescence of the components, e.g. Verbunt and $\mathrm{Zwaan} 1981$ ), the rotation period shorter, and the cool-star component(s) more active. This effect, however, is only part of proposition 7: "The presence of a companion star enhances the dynamo action in cool components of relatively close binaries not only by enforcing rapid rotation through tidal interaction, but presumably also by affecting the pattern of differential rotation resulting in an activity level well in excess of that expected from a single star of the same rotation period." Schrijver and Zwaan (1991) have given this phenomenon, called overactivity, an approximate quantification demonstrating the essential role of properties of the companion star in the activity of the other star, but the details of the physical process remain unclear. Their arguments rule out processes such as magnetic interaction between the stars and interacting winds. It appears that the dynamo efficiency in the cool binary components is increased, resulting in a stronger chromospheric and coronal activity which, apart from its high level given the associated rotation period, cannot be distinguished from that of other stars. Hence it is likely that a modified differential sotation pattern is the cause of overactivity in binaries.

\section{ACTIVITY CYCLES .}

Activity cycles are a common, although certainly not ubiquitous phenomenon among cool stars: Baliunas (1986) notes that some $60 \%$ of the 99 stars that have been monitored at Mt. Wilson during the past decades undergo (apparently) cyclic variations in the chromospheric $\mathrm{Ca} \mathrm{II} \mathrm{H}+\mathrm{K}$ emission. The relatively inactive, slow rotators generally exhibit a smooth solar-like cycle, 
while more active stars vary more sharply, possible because of the excitation of different dynamo modes or of a different dynamo mechanism (Baliunas and Vaughan 1985). Noyes et al. (1984b) present a relationship between the cycle period $P_{\text {cycle }}$ and the rotation period $P_{\text {rot }}: P_{\text {cycle }} \approx\left(P_{\text {rot }} / \tau_{\mathrm{c}}\right)^{1.25}$ for a sample of 13 slowly rotating dwarf stars, while Baliunas (1986) finds no such correlation for the entire sample of dwarfs. Maceroni et al. (1991) argue that the cycle period for main-sequence stars increases with decreasing effective temperature: $\mathrm{F}-, \mathrm{G}-$, and $\mathrm{K}$-type stars have mean cycle periods of about $5.8 \pm 1.2,7.4 \pm 0.9$, and $9.7 \pm 0.9$ years, respectively. Although they argue that stars in completely different rotational regimes (relatively narrow binaries such as the RS CVn's, cataclysmic variables and main-sequence stars) have the same distribution of cycle periods (which would eliminate a dependence of $P_{\text {cjcle }}$ on $\tau_{c}$ only, because $\tau_{c}$ differs for these stars, cf. Section III), the data samples of other than main sequence stars are too small to test for the existence of a similar colour dependence. Maceroni et al. (1990) do not find a one-parameter relationship between $P_{\text {cycle }}$ and $P_{\text {rot }}$, but their results do not exclude a role of $P_{\text {rot }}$ or $\tau_{c}$ in a multi-parameter description. Hence proposition 8: "Activity cycles tend to increase in duration with decreasing effective temperature, while the cycle period is not a function of either rotation period or convective turnover time only."

\section{ACKNOWLEDGEMENTS}

I thank R.G.M. Rutten and C. Zwaan for critically reading the manuscript, D.F. Gray and R.G.M. Rutten for their comments on the effects of evolution and activity on stellar rotation, and R.W. Noyes and D.R. Soderblom for comments. This work was supported by a fellowship grant from the Royal Netherlands Academy of Arts and Sciences.

\section{REFERENCES}

Antiochos, S.K., 1987, in Cool Stars, Stellar Systems, and the Sun, J.L. Linsky and R.E. Stencel (eds.), Springer-Verlag, p. 283.

Baliunas, S.L., 1986, in Cool Stars, Stellar Systems, and the Sun, M. Zeilik and D.M. Gibson (eds.), Springer-Verlag, p. 3.

Baliunas, S.L., Vaughan, A.H., 1985, Ann. Rev. Astron. Astrophys. 23, 379.

Basri, G., 1986, in Cool Stars, Stellar Systems, and the Sun, M. Zeilik and D.M. Gibson (eds.), Springer-Verlag, p. 184.

Basri, G., 1987a, in Cool Stars, Stellar Systems, and the Sun, J.L. Linsky and R.E. Stencel (eds.), Springer-Verlag, p. 411.

Basri, G., 1987b, Ap. J. s16, 377.

Böhm-Vitense, E., 1992, Astron. J. 1633, 608.

Durney, B.R., Latour, J., 1978, Geophys. Ap. Fluid Dyn. 9, 241.

Giampapa, M.S., Liebert, J., 1986, Ap. J. s05, 784.

Gilliland, R.L., 1985a, Ap. J. 300, 339.

Gilliland, R.L., 1985b, Ap. J. 299, 286.

Gray, D.F., 1989a, Ap. J. 347, 1021.

Gray, D.F., 1989b, in FGK Stars and T Tauri Stars, L.E. Cram and L.V. Kuhi (eds.), NASA SP-502, p.7.

Gray, D.F., 1991, in Angular Momentum Evolution of Young Stars, Catalano, S. and Stauffer, J.R. (eds.), Kluwer, The Netherlands, p. 183.

Gray, D.F., Nagel, T., 1989, Ap. J. 341, 421.

Haisch, B.M., et al., 1990, Ap. J. s61, 570. 
Haisch, B., Schmitt, J.H.M.M., Rosso, C., 1991, Ap. J. 383, L15.

Hartmann, L., Baliunas, S.L., Duncan, D.K., Noyes, R.W., 1984, Ap. J. 279, 778.

Judge, P.G., Stencel, R.E., 1991, Ap. J. 371, 357.

Lemmens, A.F.P., Rutten, R.G.M., Zwaan, C., 1992, A. \& A., in press.

Kawaler, S.D., 1987, PASP 09, 1322.

Linsky, J.L., 1992, in proceedings of symposium From EINSTEIN to $A X A F$.

Linsky, J.L., Haisch, B.M., 1979, Ap. J. 228, L27.

Maceroni, C., et al. , 1990, A. \& A. 237, 395.

MacGregor, K.B., Brenner, M., 1991, Ap. J. 876, 204.

Maeder, A., Meynet, G., 1988, A. \& A. Suppl. 76, 411.

Maeder, A., Meynet, G., 1989, A. \& A. $210,155$.

Middelkoop, F., Zwaan, C., 1981, A. \& A. 101, 26.

Noyes, R.W., 1983, in Solar and Stellar Magnetic Fields: Origins and Coronal Effects, J.O. Stenfio (ed.), Reidel, Dordrecht, p. 133.

Noyes, R.W., et al. , 1984a, Ap. J. $279,763$.

Noyes, R.W., Weiss, N.O., Vaughan, A.H., 1984b, Ap. J. $287,764$.

Parker, E.N., 1979, Cosmical Magnetic Fields; Their Origins and Their Activity, Clarendon Press, Oxford.

Pasquini, L., Brocato, E., Pallavicini, R., 1990, A. \& A. 234, 277.

Rutten, R.G.M., 1987, A. \& A. 177, 131.

Rutten, R.G.M., Schrijver, C.J., 1987, A. \& A. 177, 155.

Rutten, R.G.M., Pylyser, E., 1988, A. \& A. 191, 227.

Rutten, R.G.M., Schrijver, C.J., Lemmens, A.F.P., Zwaan, C., 1991, A. \& A. $252,203$.

Rutten, R.G.M., et al. , 1989, A. \& A. 219, 234.

Saar, S.H., 1990, in The Sun and Cool Stars: activity, magnetism, dynamos, Tuominen, I., Moss, D., and Rüdiger, G. (eds.), Springer-Verlag, p 389.

Schrijver, C.J., 1987, A. \& A. 172, 111.

Schrijver, C.J., 1991, in Mechanisms of Chromospheric and Coronal Heating, P. Ulmschneider, E.R. Priest, R. Rosner (eds.), Springer-Verlag, p. 257.

Schrijver, C.J., Dobson, A.K., Radick, R.R., 1989, Ap. J. 341, 1035.

Schrijver, C.J., Zwaan. C., 1991, A. \& A. $251,183$.

Skumanich, A., 1972, Ap. J. 171, 565.

Simon, T., Fekel Jr., F.C., 1987, Ap. J. 316, 434.

Simon, T., Drake, S.A., 1989, Ap. J. 846, 303.

Simon, T., Landsman, W:, 1991, Ap. J. 380, 200.

Soderblom, D.R., Duncan, D.K., Johnson, D.R.H., 1991, Ap. J. 375, 722.

Ulmschneider, P., Priest, E.R., Rosner, R. (eds.), 1991, Mechanisms of Chromospheric and Coronal Heating, Springer-Verlag.

Verbunt, F., Zwaan, C., 1981, A. \& A. 100, L7.

Vilhu, O., 1987, in Cool Stars, Stellar Systems, and the Sun, J.L. Linsky and R.E. Stencel (eds.), Springer-Verlag Berlin, p 110.

Vilhu, O., et al., 1989, A. \& A. 222, 179.

Walter, F.M., 1987, in Cool Stars, Stellar Systems, and the Sun, J.L. Linsky and R.E. Stencel (eds.), Springer-Verlag Berlin, p 422.

Walter, F.M., Schrijver, C.J., 1987, in in Cool Stars, Stellar Systems, and the Sun, J.L. Linsky and R.E. Stencel (eds.), Springer-Verlag, p 262.

Walter, F.M., et al. , 1988, Astron. J. 96, 297. 